\title{
A Study on Median Filter in Digital Images Based on MALTAB GUI
}

\author{
Zhang Hongmei \\ College of Computer Science and Technology \\ Inner Mongolia University For Nationalities \\ TongLiao, China \\ e-mail:Tongyu_007@163.com
}

\author{
Zhang Zhigao \\ College of Math \\ Inner Mongolia University For Nationalities \\ TongLiao, China \\ e-mail: zzgtongxin@163.com
}

\author{
Pei Zhili* \\ College of Computer Science and Technology \\ Inner Mongolia University For Nationalities \\ TongLiao, China \\ e-mail:zhilipei@sina.com \\ *corresponding author
}

\begin{abstract}
Median filter is a commonly applied technique dealing with pulse noise in image processing. The conventional method of median filtering fails to retain many of detail and marginal information and leaves images blurred or distorted when coming to noise elimination. Given the fact, another filtering technique based on self-adaption of variable windows can be adopted to keep originality of images. The article comes up with a set of algorithms and makes a design of an operation interface with the help of potentially developing GUI offerd by MATLAB. Median filtering can be operated visibly on the newly designed interface and can meet various demands of users.Simulation experiments verify its friendly usage and preferable application effect.
\end{abstract}

Keywords-median filter; self-adaptive median filtering; MATLAB GUI

\section{INTRODUCTION}

In the process of acquisition and transmission, Digital image are often disturbed by external electromagnetic, generating a pulse noise, Leading to the loss of important data $^{[1]}$. So it will have a certain impact on the subsequent segmentation, extraction and reconstruction. This noise is called Salt - Pepper Noise, which shows some black-and-white light spots on the image, and these highlights are random and superposed on each other ${ }^{[2]}$. Median filtering is often used to remove the noise. It must be related to the adjustment of the sliding window and using some algorithm to achieve adaptive median filtering, when we use median filtering to eliminate the effects of Salt - Pepper Noise. It is difficult to achieve for the normal user. Aiming to Normal user, we use the GUI MATLAB graphical interface development tool in this paper and Median filtering can be operated visibly on the newly designed interface and can meet various demands of users. Simulation experiments verify its friendly usage and preferable application effect.

\section{INTRODUCTION OF MEDIAN FILTER}

\section{(1) Traditional median filter}

Median filtering is spatial filtering algorithm based on neighborhood calculation, The response of the filter is based on the ordered queue of pixels, and the pixels are included in the image region of the filter ${ }^{[3]}$. Then the center pixel values in the image region are replaced by the intermediate values in the queue, and the mathematical expressions can be expressed as $\hat{f}(x, y)=\underset{(s, t) \in s_{s}}{\operatorname{median}}\{\mathrm{g}(\mathrm{s})\}$, where $\mathrm{g}(\mathrm{x}, \mathrm{y})$ is Noisy image and $s_{x y}$ is the sub-image of $\mathrm{g}(\mathrm{x}, \mathrm{y})$, its pixels take the $(\mathrm{x}, \mathrm{y})$ point as the center. i.e. filtering sliding window, its size is $\mathrm{m} \times \mathrm{m}^{[1]}$. For example, when $\mathrm{m}=3, \mathrm{~s}_{\mathrm{xy}}$ is a neighborhood whose size is $3 \times 3$. A series of the pixels in the neighborhood of $\{10,20,20,20,15,25,25,20,100\}, \quad$ ascending order is $\{10,15,20,20,20,20,25,25,100\}$, median is 20 ,which is the fifth value in the sequence. In fact, the $\mathrm{m} \times \mathrm{m}$ median filter is used to remove the isolated pixels, which is brighter or darker than those of the neighborhood pixels, and these pixels are forced to be the median gray of neighborhood [4]. Neighborhood processing is shown in Fig. 1.

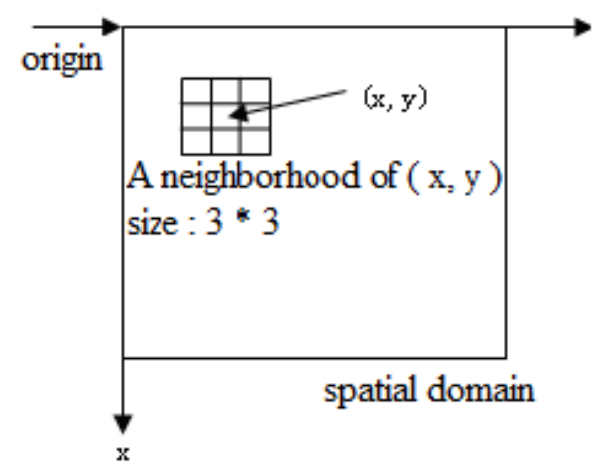

Fig.1. Neighborhood processing sketch 
From the neighborhood calculation process shown in figure 1, we can see that the filter performs median filtering operations on all pixels sequentially from the origin. This method does not consider the pixels that are not contaminated by noise, and all will be converted into a median pixel, even though it can suppress the impulse noise, the effect of noise reduction is achieved. The direct consequence of this method is that the image is easy to lose many edges and details, so that the image becomes blurred or distorted.

The main code is as follows:

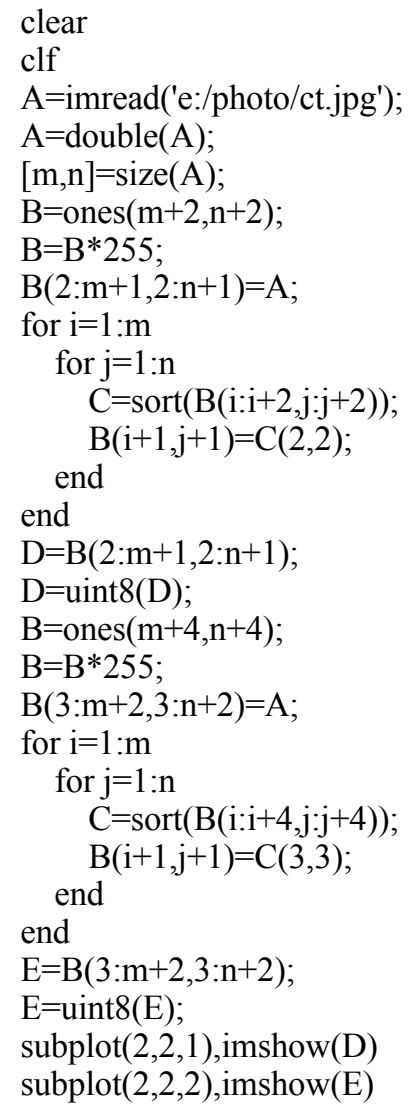

\section{(2) Adaptive median filter}

Aiming at the defects of the traditional median filter, some scholars propose the adaptive median filtering method. Different from the traditional median filter, the adaptive median filter is automatically based on certain conditions to change the size of the sliding window $\mathrm{s}_{\mathrm{xy}}{ }^{[5]}$. In the reference [1], the algorithm is summarized as follows:

Let $z_{\min }$ be the minimum values of $\mathrm{s}_{\mathrm{xy}}$ and $z_{\max }$ be the maximum values of $\mathrm{s}_{\mathrm{xy}}$. Let $z_{\text {med }}$ be the median values of $\mathrm{s}_{\mathrm{xy}}$ and $z_{x y}$ be the gray value of Pixel point(x, y). $\mathrm{m}$ is the size of median filter window. $S_{\max }$ is the maximum sliding window size allowed by the adaptive filter. Algorithm chart for selfadaptive median filtering is shown in Fig. 2.

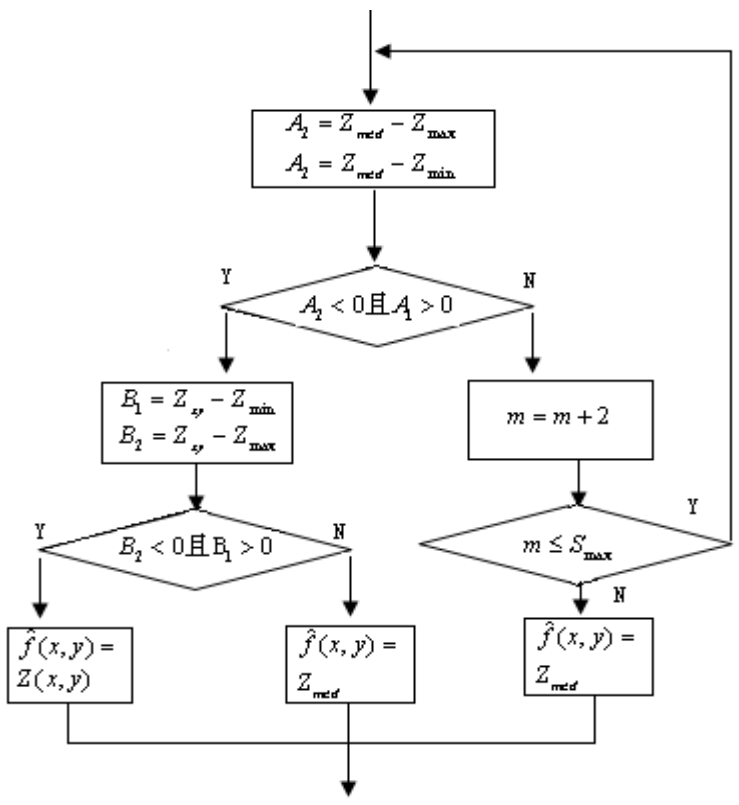

Fig. 2. Algorithm chart for self-adaptive median filtering

The algorithm adaptively changes the size of the filter window, according to the test results that whether $z_{x y}$ and $z_{\text {med }}$ are the impulse noise points. It avoids the disadvantages of the traditional median filter that all the pixels are replaced with the median value, reducing the unnecessary losses of the image details effectively.

\section{REALIZATION OF VISUAL MEDIAN FILTER GRAPHICS INTERFACE}

Visualization of digital image processing is a developing trend of image processing technology [5]. In this paper, the GUIDE (GUI) is used to design the graphical user interface, which is the development environment of MATLAB. The function code is written as a callback function ${ }^{[6]}$. The function codes written by myself could be used as callback functions of the interface control, the two kinds of median filter are realized in the above section ${ }^{[7]}$. Operation interface for users is shown in Fig.3.

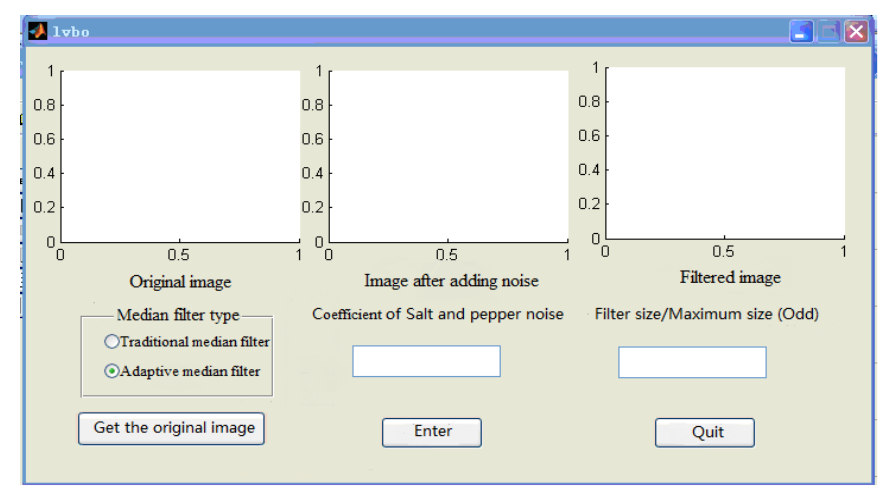

Fig.3. Operation interface for users

Use the getting the original image command button to display a original image on the axis 1 , and then enter a number 
that must be positive number less than 1 in the edit box, which is called the coefficient of adding salt and pepper noise, click the OK button. After adding noise, the image will be displayed on the axis 2. Now come the question of size of the median filter window, the size number should be an odd number greater than 3 , and then choose the type of median filter, the filtered image will be displayed on the axis 3 .

The main code is as follows :

clear

clf

$\mathrm{I}=$ imread('e:/photo/ct.jpg');

Ig=rgb2gray $(\mathrm{I})$;

Inoise=imnoise (Ig,'salt \& pepper',0.2);

subplot(2,2,1),imshow(Ig);

xlabel('a. Original gray scale image ');

subplot(2,2,2),imshow(Inoise);

xlabel('b. Images contaminated by noise ');

$[\mathrm{Im}, \mathrm{In}]=\operatorname{size}($ Inoise);

nmin $=3$;

$\operatorname{nmax}=9$;

$\mathrm{Imf}=$ Inoise;

I_ex $=[\operatorname{zeros}((\operatorname{nmax}-1) / 2, \operatorname{In}+(\operatorname{nmax}-1))$;

zeros(Im,(nmax-1)/2),Inoise,zeros(Im,(nmax-1)/2);

zeros $(($ nmax-1)/2,In+(nmax-1))];

for $x=1: \operatorname{Im}$

for $y=1:$ In

for $\mathrm{n}=\mathrm{nmin}: 2: \mathrm{nmax}$

$\mathrm{Sxy}=\mathrm{I} \_\mathrm{ex}(\mathrm{x}+(\mathrm{nmax}-1) / 2-(\mathrm{n}-1) / 2: \mathrm{x}+(\mathrm{nmax}-$

$1) / 2+(n-1) / 2, y+(n m a x-1) / 2-(n-1) / 2: y+(n m a x-1) / 2+(n-1) / 2)$;

$\operatorname{Smax}=\max (\max (\operatorname{Sxy}))$

$\operatorname{Smin}=\min (\min (\operatorname{Sxy}))$;

Smed $=$ median $($ median $($ Sxy $))$;

if Smed $>$ Smin \& \& Smed $<$ Smax

if $\operatorname{Imf}(\mathrm{x}, \mathrm{y})<=\operatorname{Smin} \| \operatorname{Imf}(\mathrm{x}, \mathrm{y})>=\operatorname{Smax}$

$$
\text { end }
$$$$
\operatorname{Imf}(\mathrm{x}, \mathrm{y})=\text { Smed; }
$$

break

end

end

end

$\operatorname{Imf}(\mathrm{x}, \mathrm{y})=$ Smed;

end

subplot(2,2,3),imshow(Imf);

Imf1 $=$ medfilt 2 (Inoise, $[3,3])$;

$\operatorname{Imf} 2=$ medfilt $2(\operatorname{Imf} 1,[3,3])$;

subplot(2,2,4),imshow(Imf2);

Effect of operation is shown in Fig.4.

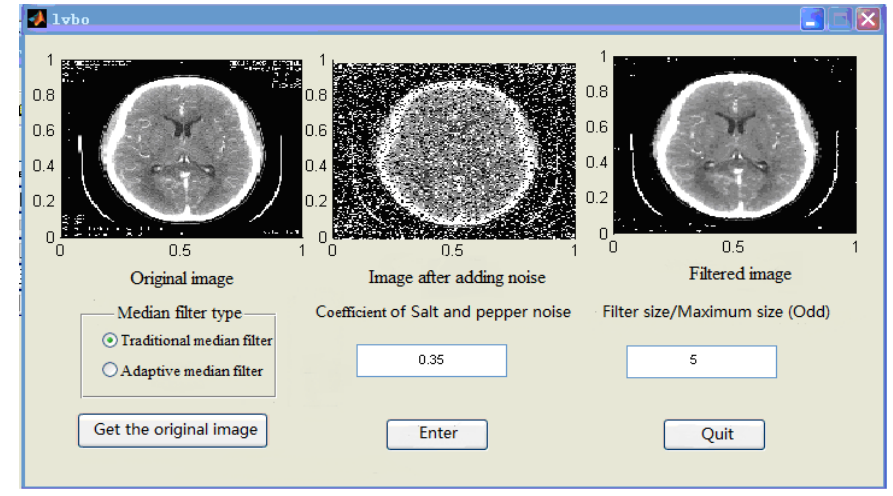

Fig.4. Effect of operation

\section{SIMULATION EXPERIMENTS}

This paper selects a frame of medical CT image, adding $35 \%$ of the impulse noise. The system is used to reconstruct the noisy image, where traditional median filter and adaptive median filter are adopted respectively. The simulation results of two kinds of filtering are shown in Fig.5.

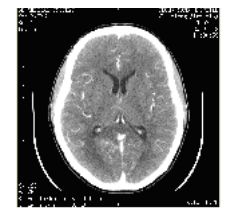

Original CT image

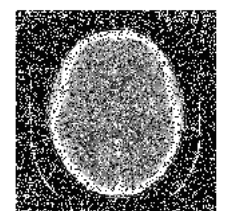

Image after adding nois

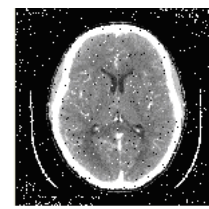

$3 \times 3$ Median filter

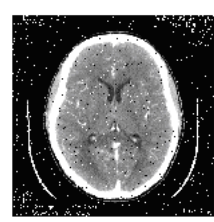

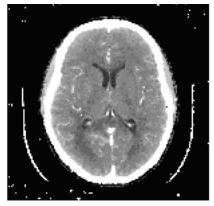

$5 \times 5$ Median filter

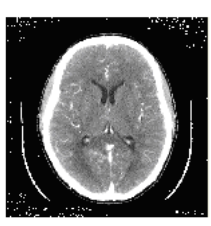

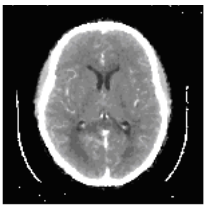

$7 \times 7$ Median filter

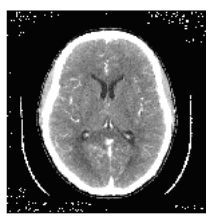

Fig.5.CT median filtering sketch and self-adaptive median filtering sketch

The results of the Image filtering simulation experiment show that the two filtering methods used in this paper can achieve better filtering effect. When filtering window increases, the image after median filtering will become blurred gradually, the direct consequence of this method is that the image is easy to lose many edges and details, so that the image becomes distorted ${ }^{[8]}$. Using the adaptive median filter can better retain some of the image edge information, reduce the distortion degree, can achieve better filtering effect than median filtering ${ }^{[9]}$.

\section{PERORATION}

In this paper, we summarize and realize two algorithms of the traditional median filter and the adaptive median filter. The GUIDE (GUI) of the development environment of MATLAB is used to design and develop the graphical user interface, which can achieve the image median filter. Through the simulation experiment of median filter and adaptive median filter in the system, the better experimental results are achieved. 


\section{ACKNOWLEDGMENT}

This work was financially supported by the National Natural Science Foundation of China (61163034, 61373067), the Grassland Excellent Talents Project of Inner Mongolia Autonomous Region (2013), the supported By Program for Young Talents of Science and Technology in Universities of Inner Mongolia Autonomous Region(NJYT-14-A09), the Inner Mongolia Natural Science Foundation(2013MS0911), the 321 Talents Project the two level of Inner Mongolia Autonomous Region(2010), the Inner Mongolia talent development fund(2011), and the Scientific Research Foundation of Inner Mongolia University For Nationalities(NMDYB15011)

\section{REFERENCES}

[1] Rafael C. Gonzalez, Richard E. Woods. Digital Image Processing(Third Edition)[M].Ruan Qiuqi, translation. Beijing: Publishing House Of Electronics Industry,2011.6:72-77.
[2] HU Qiong,WANG Ronggui,HU Weiwei,YANG Wanting. Color Image Enhancement Based on Histogram Segmentation[J]. Journal of Image and Graphics,2009,14(9):1776-1781.

[3] Zheng Yongguo. Research and Implementation on image enhancement methods [D]. Shandong University of Science and Technology,2005.

[4] LI Guanzhang,LUO Wusheng,LI Pei. Color Image Enhancement Based on Visual Characteristics of Human Eyes[J]. Opto-Electronic Engineering,2009,36(11):92-95.Yoon Byoungwoo,Song Woojin.Image Contrast Enhancement Based on the Teneralized Histogram[J].Journal of Electronic Imaging,2007,16(3):033005-033005-8.

[5] Zhang Yi,Liu Xu,Li Haifeng. Adaptive Image Histogram Equalization Algorithm [J].Journal of ZheJiang University (Engineering Science) .2007,Vol. 41 No.4.

[6] Ruan Qiuqi. Digital Image Processing [M]. Beijing: Publishing House Of Electronics Industry,2001.

[7] Castleman Kenneth R. Digital Image Processing[M].Zhu Zhigang, translation.Beijing: Publishing House Of Electronics Industry,1998.

[8] Wang Qiwei. Research on image histogram feature and its application[D].University of Science and Technology of China,2014.

[9] GONZALEZ RC,WOODS R E,EDDIN S S L. Digital Image Processing using MATLAB [M].Beijing: Publishing House Of Electronics Industry,2004. 